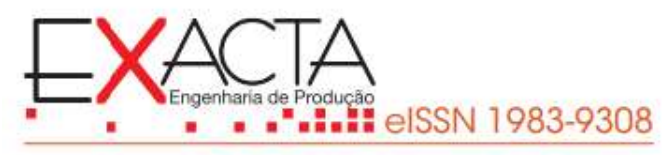

https://doi.org/10.5585/ExactaEP.v18n3.10928

\title{
Eficiência dos serviços de segurança pública no Brasil: \\ uma análise por envoltória de dados
}

\author{
Efficiency of public security services in Brazil: \\ data envelopment analysis
}

\author{
Isabel Silva Almeida ${ }^{1}$
}

Anselmo Ramalho Pitombeira Neto ${ }^{2}$

\section{${ }^{1}$ Universidade Federal do Ceará Bio: Engenheira de Produção Mecânica pela Universidade Federal do Ceará. Analista de Processos na Unimed Fortaleza, Ceará, Brasil. isalme8@gmail.com \\ ${ }^{2}$ Universidade Federal do Ceará} Bio: Doutor em Engenharia de Transportes. Chefe do Departamento de Engenharia de Produção da UFC e líder do Grupo de Pesquisa $\mathrm{OPL}$ - Pesquisa Operacional em Produção e Logística. Integrante do corpo docente do Mestrado em Modelagem e Métodos Quantitativos, Departamento de Estatística e Matemática Aplicada, UFC. , Campus do Pici, Fortaleza, Brasil. anselmo.pitombeira@ufc.br

\begin{abstract}
Resumo
Este estudo propõe uma avaliação da segurança pública do Brasil através da análise por envoltória de dados. Assumindo-se os 27 estados federativos como as unidades tomadoras de decisão, foram utilizados como inputs as despesas totais com segurança e o efetivo policial total de cada estado, e como output a razão entre o número de habitantes e o número de CVLIs (Crimes Violentos Letais Intencionais), definida como uma "taxa de segurança". Os dados foram obtidos do Anuário Brasileiro de Segurança Pública, com informações referentes ao ano de 2016. Os resultados indicaram 6 estados na fronteira de eficiência: Santa Catarina, Piauí, Roraima, São Paulo, Rio Grande do Sul e Maranhão. Do total, 33,33\% dos estados apresentaram baixa eficiência, 25,93\% deles eficiência moderada e 18,52\% deles eficiência alta. Em um cenário de escassez de recursos, um indicador científico das ineficiências e possibilidades de realocação destes recursos é extremamente pertinente.
\end{abstract}

Palavras-chave: Análise de eficiência. Análise por envoltória de dados. Segurança pública.

\begin{abstract}
This study proposes an evaluation of the Brazilian public security through data envelopment analysis. Assuming the 27 states as the decision-making units, the total expenditures with security and the total police force of each state were used as inputs, and the ratio between the inhabitants / VILC (Violent Intentional Lethal Crimes), defined as a "security rate." The data were obtained from the Brazilian Yearbook of Public Safety, with information referring to the year 2016. The results indicated 6 states at the efficiency frontier: Santa Catarina, Piauí, Roraima, São Paulo, Rio Grande do Sul and Maranhão. Of the total, 33.33\% of the states presented low efficiency, $25.93 \%$ of them moderate efficiency and $18.52 \%$ of them high efficiency. In a resource scarcity scenario, a scientific estimate of the inefficiencies and possibilities for reallocating these resources is extremely pertinent.
\end{abstract}

Keywords: Efficiency analysis. Data envelopment analysis. Public security. 


\section{Introdução}

A segurança pública no Brasil ganhou enorme visibilidade e nunca esteve tão presente nos debates tanto de especialistas quanto do público em geral. Os problemas relacionados ao aumento das taxas de criminalidade e aumento da sensação de insegurança representam obstáculos para a consolidação política da democracia no país (Observatório de Segurança Pública, 2018).

Como os demais serviços públicos, a segurança deve-se orientar pelo princípio da eficiência, ou seja, proporcionar o melhor serviço possível com o menor dispêndio de recursos. A eficiência da segurança, além de garantir a economicidade dos serviços, implica na contenção dos efeitos nocivos da criminalidade contra a pessoa ou contra o patrimônio, tendo em vista que a contenção do crime proporciona melhoria do ambiente econômico e social (Lima \& Marinho, 2016).

Uma das técnicas mais utilizadas para a análise da eficiência de unidades semelhantes é a análise por envoltória de dados (Data Envelopment Analysis - DEA) (Charnes, Cooper, \& Rhodes, 1978; Emrouznejad, Banker, Lopes, \& Almeida, 2013). Modelos DEA são rotineiramente empregados na avaliação do setor privado, como na análise do desempenho de bancos e instituições financeiras, assim como na avaliação do setor público, como hospitais e sistemas de saúde, ou escolas e universidades. A vantagem desta técnica é que ela acomoda múltiplos inputs e outputs para medir as eficiências relativas de um conjunto de unidades tomadoras de decisão (decision making units DMUs). A DEA não requer que os valores das variáveis sejam monetários para devolver um diagnóstico de eficiência, o que é uma vantagem significativa para o setor público, onde estes valores nem sempre são explícitos ou estão disponíveis. As comparações entre os diferentes níveis de eficiência das unidades produtivas podem ser usadas para se proporem mudanças e melhorias na administração de serviços públicos.

O presente trabalho tem por objetivo estimar os escores de eficiência dos serviços de segurança pública dos 27 estados brasileiros através da DEA, de forma a identificar as unidades eficientes, classificá-las segundo suas eficiências e projetar metas para as unidades de forma a se tornarem eficientes. Para isso é aplicado um modelo DEA do tipo BCC (modelo de Banker, Charnes e Cooper) com uso dos dados do Anuário Brasileiro de Segurança Pública, com informações referentes ao ano de 2016.

Este artigo está dividido nas seguintes seções: Na seção 2 apresenta-se uma breve revisão bibliográfica; na Seção 3 é detalhado o método utilizado; na Seção 4 são apresentados os resultados; finalmente conclui-se o trabalho na Seção 5 (Conclusão). 


\section{Revisão bibliográfica}

\subsection{Análise por envoltória de dados - DEA}

Diferentemente das técnicas tradicionais de benchmarking em que empresários observam, comparam e identificam as melhores práticas, a DEA é orientada para a análise de dados empíricos através de um modelo matemático, minimizando a interferência de suposições que podem causar distorções nos resultados. (Colin, 2007).

A função que relaciona a máxima quantidade de produtos, ou outputs, que podem ser gerados por uma dada quantidade de recursos, ou inputs, é chamada "função de produção". A função de produção mapeia as entradas em relação às saídas e é uma representação matemática de como uma empresa pode utilizar recursos do seu processo produtivo (Fioretti, 2008), isto é, indica o mais alto valor de outputs que a empresa pode produzir para cada combinação específica de inputs (Besanko \& Braeutigam, 2011). Tomando-se $Q$ como a quantidade de outputs e $L$ como a quantidade de inputs, a Figura 1 ilustra uma função de produção.

Figura 1 - Curva da função de produção

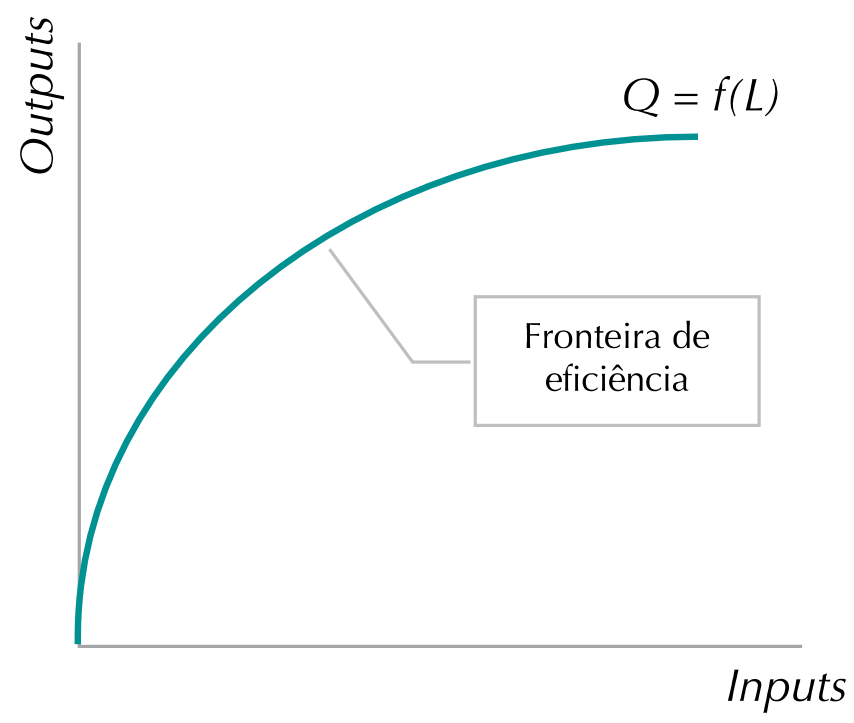

Fonte: Adaptado de Besanko e Braeutigam (2011).

A curva $Q=f(L)$ delimita o máximo que pode ser produzido para cada nível de inputs, e a área abaixo da curva corresponde ao conjunto viável de produção (Mello, Meza, Gomes, \& Neto, 2005), ou todas as combinações possíveis de inputs e outputs. Uma vez que a curva representa a produção máxima possível, qualquer DMU que tenha produção abaixo da curva está subutilizando seus recursos, enquanto DMUs situadas exatamente na curva são eficientes. Por este motivo, a curva é também chamada de "fronteira de eficiência".

Os métodos não-paramétricos para cálculo da eficiência, entre os quais está inclusa a DEA, não assumem uma forma específica para a função de produção. Desta forma, consideram que o 
máximo que pode ser produzido é obtido por meio da observação das unidades mais produtivas (Mello et al., 2005). Em outras palavras, a DEA estima a fronteira de eficiência com base no máximo desempenho observado entre as DMUs, conforme a Figura 2.

Figura 2 - Fronteira de eficiência estimada pela DEA (retornos variáveis de escala)

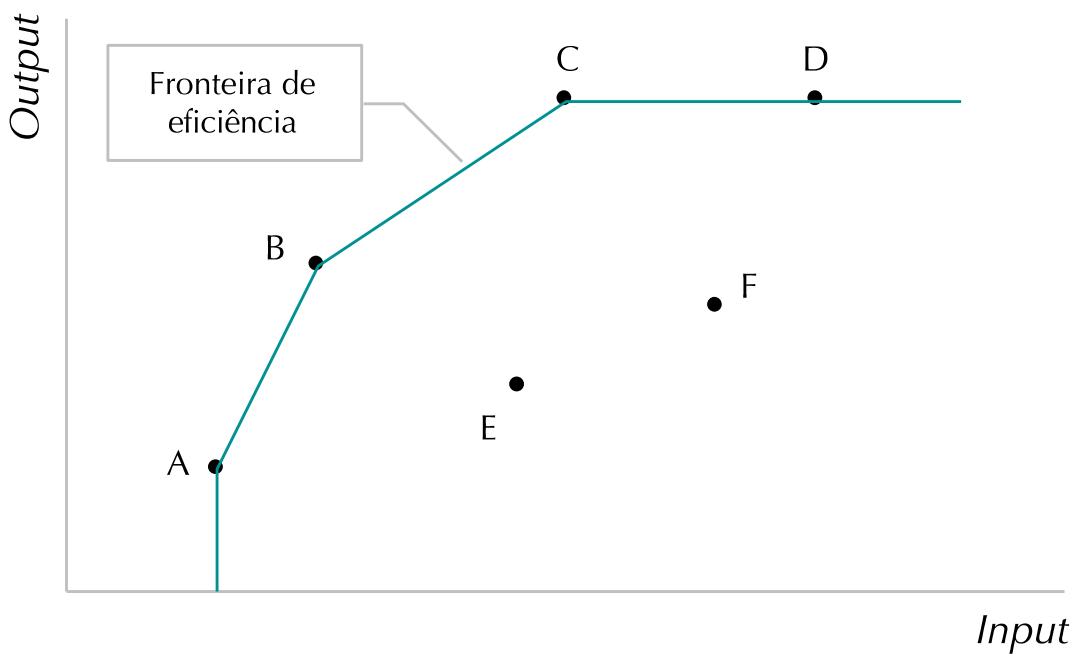

Fonte: Os autores.

Na Figura 2, percebe-se que a fronteira de eficiência é formada pelas DMUs $A, B, C$ e $D$, que demonstraram melhor relação entre o consumo de inputs e produção de outputs (eficiência 100\%), enquanto as DMUs E e F estão abaixo da curva, de forma que são consideradas ineficientes.

Existem diversos modelos DEA. Os mais utilizados são o modelo CCR (Charnes, Cooper, \& Rhodes, 1978) e BCC (Banker, Charnes, \& Cooper, 1984). O modelo CCR assume retornos constantes de escala, enquanto o modelo BCC assume retornos variáveis de escala. Adicionalmente, os modelos podem ser "orientados a inputs", quando os outputs são mantidos constantes e a eficiência é estimada com base na minimização dos inputs, ou "orientado a outputs", quando os inputs são mantidos constantes e a eficiência é estimada com base na maximização dos outputs. Mais detalhes quanto à metodologia DEA podem ser encontrados em (Mello et al., 2005).

\subsection{Aplicação de DEA à segurança pública no Brasil}

Em se tratando da segurança pública brasileira, estudos empíricos que analisam a eficiência do uso dos recursos através da DEA são escassos. Na Tabela 1 são apresentados os principais estudos desenvolvidos no Brasil sobre o tema até o presente, comentadas nos parágrafos a seguir. 
Tabela 1 - Estudos que utilizam a DEA com método de avaliação da eficiência dos serviços de segurança pública no Brasil

\begin{tabular}{|c|c|c|c|c|}
\hline Autor & DMUs & Inputs & Outputs & Resultados \\
\hline $\begin{array}{l}\text { Fernandes } \\
(2016)\end{array}$ & $\begin{array}{l}\text { Todos os } \\
\text { estados com } \\
\text { exceção de } \\
\text { BA, PB, CE e } \\
\text { RO }\end{array}$ & $\begin{array}{l}\text { Despesas totais } \\
\text { com segurança } \\
\text { pública per capita }\end{array}$ & $\begin{array}{l}\text { Inverso das taxas de } \\
\text { Homicídios, Latrocínios, } \\
\text { Roubos, Tráfico e Estupros / } \\
100 \text { mil habitantes } \\
\text { No. de presos / } 100 \text { mil } \\
\text { habitantes com mais de } 18 \\
\text { anos }\end{array}$ & $\begin{array}{l}\text { Em 2014, 56\% dos estados } \\
\text { apresentaram ineficiência } \\
\text { nos gastos com segurança } \\
\text { pública. Os estados } \\
\text { identificados como } \\
\text { eficientes foram SP, AC, SC, } \\
\text { AP, RN, PI, ES, PR, MG e } \\
\text { MS. }\end{array}$ \\
\hline Paulo (2016) & $\begin{array}{l}\text { Todos os } \\
\text { estados }\end{array}$ & $\begin{array}{l}\text { Gastos com } \\
\text { Policiamento, } \\
\text { Defesa Civil, } \\
\text { Informação e } \\
\text { Inteligência, } \\
\text { Demais } \\
\text { Subfunções }\end{array}$ & $\begin{array}{l}\text { Número de Homicídios, } \\
\text { Crimes letais intencionais, } \\
\text { Outros crimes letais, Crimes } \\
\text { violentos letais } \\
\text { intencionais, Crimes } \\
\text { violentos não letais } \\
\text { intencionais } \\
\text { Número de leis especiais }\end{array}$ & $\begin{array}{l}\text { Em } 2012 \text { e 2013, 85\% e } \\
78 \% \text { dos estados } \\
\text { apresentaram ineficiência, } \\
\text { respectivamente. Em 2012, } \\
\text { AP, MS, PE e SP foram } \\
\text { considerados eficientes, e } \\
\text { em 2013, AP, MS, PE, SP, RJ } \\
\text { e PI. }\end{array}$ \\
\hline $\begin{array}{l}\text { Schull, } \\
\text { Feitósa e } \\
\text { Hein (2014) }\end{array}$ & $\begin{array}{l}\text { Todos os } \\
\text { estados com } \\
\text { exceção de } \\
\text { AP, PI, RN e } \\
\text { RR }\end{array}$ & $\begin{array}{l}\text { Despesas totais } \\
\text { com segurança } \\
\text { pública }\end{array}$ & $\begin{array}{l}\text { Taxas de homicídios, } \\
\text { Latrocínios, Homicídios } \\
\text { culposos de trânsito, } \\
\text { Tráfico e Estupros / } 100 \text { mil } \\
\text { habitantes }\end{array}$ & $\begin{array}{l}\text { Em 2011, } 48 \% \text { dos estados } \\
\text { apresentaram ineficiência } \\
\text { nos gastos com segurança } \\
\text { pública. Os estados } \\
\text { identificados como } \\
\text { eficientes foram AC, AL, CE, } \\
\text { DF, ES, MA, MT, MG, PA, } \\
\text { PR, RS, RO. }\end{array}$ \\
\hline
\end{tabular}

Fonte: Os autores.

Fernandes (2016) avalia a eficiência relativa dos serviços de segurança de 23 estados no ano de 2014. Os estados da Bahia, Paraíba, Ceará e Roraima foram excluídos da amostra por não apresentarem dados completos naquele ano. Utilizando-se as despesas com segurança per capita como input e o inverso de diversas taxas criminais, além do inverso da taxa de presos a cada 100 mil habitantes com mais de 18 anos, como outputs, o autor conclui que 56\% das unidades federativas gasta seus recursos financeiros de maneira ineficiente. Destas, 8 apresentam eficiências menores que 25\%, evidenciando-se o enorme desperdício dos recursos públicos. Uma limitação do trabalho ocorre no uso inadequado de razões. Apesar de o uso de razões como variáveis não ser incomum, especialmente em modelos da área da saúde e financeira, ele fere o axioma da convexidade da DEA, e requer tratamento adequado conforme será discutido na Seção 3. Outra limitação está na alta relação entre o número de variáveis e o número de DMUs, uma vez que um número muito alto de variáveis pode "achatar" os dados, fazendo com que ela enxergue mais unidades eficientes do que realmente o são (Adler \& Yazhemsky, 2010). Neste caso, a redução do número de estados contemplados pelo modelo (DMUs), aliada ao uso de 1 input e 6 outputs, pode explicar o porquê de quase metade (44\%) dos estados terem sido considerados completamente eficientes. 
Paulo (2016) calculou a eficiência dos serviços de segurança para todas as 27 unidades federativas nos anos de 2012 e 2013. Utilizando-se 4 classes de dispêndio com segurança como inputs e os números absolutos de 6 tipos de crimes como outputs, a autora encontrou que $85 \%$ dos estados consumia seus recursos financeiros de forma ineficiente em 2012, e 78\% em 2013. Notavelmente, todos os estados identificados como eficientes em 2012 mantiveram-se como tal no ano seguinte, apontando para a manutenção do padrão de serviço no período. Apesar de o modelo de Paulo (2016) apresentar eficiências mais discriminadas, a grande quantidade de variáveis utilizadas em relação à quantidade de DMUs também pode diminuir a confiabilidade de seus resultados. Além disto, nota-se uma inadequação na definição de algumas variáveis. Por exemplo, enquanto a DEA calcula as eficiências assumindo que um aumento dos inputs acarretará em aumentos nos outputs, as variáveis selecionadas por Paulo (2016) apresentam comportamento oposto: espera-se que um aumento dos gastos com segurança pública (inputs) reduza o número de crimes praticados (outputs). Os outputs, neste caso, deveriam ter sido adaptados para uma forma que crescesse junto com os inputs, como por exemplo o inverso das taxas criminais, conforme adaptado por Fernandes (2016).

Por fim, o trabalho de Schull, Feitosa e Hein (2014) avaliou a eficiência de 23 dos estados em relação aos gastos com segurança pública, afirmando que $48 \%$ deles operavam de forma ineficiente em 2011. O estudo apresenta falhas semelhantes às discutidas para os outros autores: o alto número de variáveis em relação à quantidade de DMUs, o uso de razões (taxas) como variáveis, e a relação inversa entre acréscimos de inputs e outputs. No entanto, uma característica que destaca este estudo dos demais é que, após o cálculo das eficiências, as autoras indicam metas para que os estados alcancem a eficiência baseados na projeção da DEA.

Avaliando-se o corpo de pesquisa disponível para o tema, percebe-se que há um grande consenso sobre as variáveis adequadas para calcularem-se as eficiências dos serviços de segurança em cada estado: os investimentos na área como inputs, e alguma forma das taxas criminais como outputs. Este trabalho busca superar as limitações identificadas nos estudos anteriores quanto ao número de variáveis e uso de variáveis-razão. Ademais, em nenhum dos casos os autores discutiram as DMUs que poderiam ser usadas como benchmarks para os estados ineficientes, uma informação secundária calculada pela DEA considerada um dos seus pontos fortes e um dos motivadores para o uso da técnica. Neste trabalho, além de identificar as DMUs eficientes, também são estabelecidas metas para as DMUs ineficientes.

\section{Metodologia}

\subsection{Escolha das variáveis e orientação do modelo}

Os dados utilizados para desenvolvimento do modelo DEA foram retirados da edição de 2017 do Anuário Brasileiro de Segurança Pública - ABSP, com dados referentes ao ano de 2016. O ABSP 
compila e analisa dados de registros policiais sobre criminalidade, informações sobre o sistema prisional e gastos com segurança pública, entre outros recortes introduzidos a cada edição (Fórum Brasileiro de Segurança Pública, 2017).

No ABSP 2017 os estados são divididos conforme Quadro 2, de acordo com a qualidade estimada dos registros estatísticos oficiais de segurança pública:

Tabela 2 - Grupos de estados segundo qualidade estimada dos dados

\begin{tabular}{|c|c|c|}
\hline Grupo & Qualidade de informações & Estados \\
\hline 1 & Maior qualidade das informações & $\begin{array}{c}\text { AL, AM, CE, ES, MG, MT, } \\
\text { PA, PB, PE, PI, PR, RJ, RN, } \\
\text { SC }\end{array}$ \\
\hline 2 & Qualidade intermediária das informações & $\begin{array}{c}A C, D F, G O, M A, M S, R S \\
S E, S P\end{array}$ \\
\hline 3 & Menor qualidade das informações & $A P, R O, R R, T O$ \\
\hline 4 & $\begin{array}{c}\text { Não há como atestar a qualidade dos } \\
\text { dados, pois a UF optou por não responder } \\
\text { o questionário de avaliação }\end{array}$ & $\mathrm{BA}$ \\
\hline
\end{tabular}

A classificação indicada no Quadro 2 foi obtida através da aplicação de formulários respondido pelos gestores estaduais do Sistema Nacional de Estatísticas de Segurança Pública e Justiça Criminal (SINESPJC), responsáveis pela produção das estatísticas pelas unidades federativas - Ufs (Fórum Brasileiro de Segurança Pública, 2017).

A Tabela 3 a seguir resume as variáveis escolhidas para este estudo, discutidas em seguida.

Tabela 3 - Definição das variáveis do modelo DEA

\begin{tabular}{|c|c|c|c|}
\hline Variável & Nome & Fonte de dados & Cálculo \\
\hline Input 1 & $\begin{array}{l}\text { Despesas } \\
\text { Totais }\end{array}$ & $\begin{array}{l}\text { ABSP 2017, Tabela } 26 \text { - Despesas } \\
\text { realizadas com a Função Segurança } \\
\text { Pública, por Subfunção }\end{array}$ & $\begin{array}{c}\text { Despesas realizadas com a Função } \\
\text { Segurança Pública (Total) }\end{array}$ \\
\hline Input 2 & $\begin{array}{l}\text { Efetivo } \\
\text { Total }\end{array}$ & $\begin{array}{c}\text { ABSP 2017, Tabela } 25 \text { - Efetivo fixado e } \\
\text { existente das Polícias Militar e Civis }\end{array}$ & $\begin{array}{c}\text { Efetivo existente da Polícia Militar + } \\
\text { Efetivo existente da Polícia Civil }\end{array}$ \\
\hline Output 1 & $\begin{array}{l}\text { Taxa de } \\
\text { Segurança }\end{array}$ & $\begin{array}{l}\text { ABSP 2017, Tabela } 01 \text { - Mortes violentas } \\
\text { intencionais; } \\
\text { IBGE, População residente enviada ao } \\
\text { Tribunal de Contas da União - 2001- } \\
2017\end{array}$ & $\begin{array}{l}\text { Razão entre a população e número de } \\
\text { crimes CVLI (Homicídio Doloso + } \\
\text { Latrocínio + Lesão Corporal Seguida de } \\
\text { Morte) do estado }\left(\frac{\text { População }}{\text { No.CVLI }}\right)\end{array}$ \\
\hline
\end{tabular}

Fonte: Os autores. 
Das informações disponibilizadas pelo ABSP, foram escolhidos como inputs o item "despesas totais", encontrado na tabela "Despesas realizadas com a Função Segurança Pública, por Subfunções" como a soma das despesas com policiamento, defesa civil, informação e inteligência, e demais subfunções; e o item "efetivo total", calculado a partir da tabela "Efetivo fixado e existente das Polícias Militares e Civis" somando-se os efetivos existentes (isto é, excluindo-se Corpo de Bombeiros) militares e civis. Para os estados onde os dados do efetivo de 2016 ainda não haviam sido disponibilizados, os valores foram estimados por uma suavização exponencial simples dos dados de 2010-2015, dada por (Pellegrini, 2000):

$$
\hat{z}_{t+1}=\alpha z_{t}+(1-\alpha) \hat{z}_{t}
$$

em que $\hat{z}_{t+1}$ é a previsão do efetivo no tempot+1, feita no período atual $t ; \alpha$ é a constante de suavização, assumindo valores entre 0 e $1 ; z_{t}$ é o efetivo observado na série temporal para o tempo $t$; e $\hat{z}_{t}$ é o valor da previsão feita para o tempo $t$. Adotou-se $\alpha=0,75$ após ajuste aos dados referentes à série histórica de 2010-2015, e então foi feita a previsão do efetivo para 2016.

Como output, decidiu-se incluir apenas os "crimes violentos letais intencionais" (CVLI), definidos como a soma dos homicídios dolosos, latrocínios e lesões corporais seguidas de morte (Fórum Brasileiro de Segurança Pública, 2017). Apesar de existirem outros tipos de crimes relevantes, os CVLI são aqueles com intenção e resultado mais graves, podendo assim representar índices gerais de violência. Adicionalmente, é importante preservar um número pequeno de variáveis que sejam representativas no modelo, já que uma grande quantidade destas pode interferir com a capacidade da DEA de distinguir as DMUs mais eficientes (Adler \& Yazhemsky, 2010).

Como a DEA assume que um incremento nos inputs deve provocar um incremento nos outputs, não seria adequado simplesmente utilizar o número absoluto de CVLI como output, pois se espera que este diminua com o aumento dos inputs. Deve-se, então, pensar em como mensurar a segurança em relação aos CVLI levando-se em consideração que ela deve aumentar com acréscimos nos inputs. Uma solução é utilizar como output a razão entre o número de habitantes de um estado e seu respectivo número de ocorrências de CVLI: quanto maior o número de habitantes para cada CVLI, menos CVLI estão ocorrendo naquele estado, e, portanto, mais seguro ele é. Para produzir esta razão, foram utilizadas as estimativas populacionais do IBGE para cada UF em 2016.

Finalmente, assumindo-se que um aumento nas despesas na área de segurança e efetivo policial (inputs 1 e 2) seria improvável dado o histórico nacional de escassez de recursos públicos, e que em contrapartida, uma redução poderia ser considerada antiética visto que nem mesmo os esforços atuais são capazes de gerar segurança, optou-se por orientar o modelo DEA a outputs, de forma que este indique onde é possível maximizar a eficiência mantendo-se inalterados os inputs. 


\subsection{Considerações acerca do uso de um output-razão na DEA}

Em alguns casos encontrados na literatura, ao invés de variáveis absolutas, são utilizadas variáveis que são resultado de uma razão como input (input-razão) e/ou output (output-razão), o que pode ser necessário para refletir adequadamente a função produção ou simplesmente por causa da natureza dos dados disponíveis. Na área da saúde, por exemplo, muitos dos indicadores de desempenho são expressos em forma de razão, como porcentagem de gastos em relação ao lucro bruto, taxa de altas hospitalares por 100.000 habitantes, ou mesmo o número anual de faltas por doença por funcionário. Hollingsworth e Smith (2003) afirmam que para estes casos a formulação do modelo CCR não é adequada, demonstrando que o modelo BCC deveria ser usado na presença de um input ou output-razão.

Na modelagem matemática da DEA, um dos pressupostos é que a fronteira de eficiência é convexa (axioma da convexidade), isto é, todas as combinações convexas possíveis de possibilidades de produção encontram-se contidas na fronteira de eficiência. No entanto, Emrouznejad e Amin (2009) afirmam que o uso de inputs ou outputs-razão viola o axioma da convexidade mesmo com uso do modelo BCC.

Para superar este problema, uma das soluções apresentadas pelos autores é desagregar a variável-razão em seu numerador e denominador, designando-os como inputs ou outputs adicionais dependendo do tipo e orientação do modelo. Quanto ao tipo de modelo mais adequado para avaliação da segurança pública, além da recomendação de Hollingsworth e Smith (2003) para uso do BCC na presença de variáveis-razão, entende-se que este é mesmo o tipo mais apropriado já que se espera que os retornos de escala não sejam sempre constantes para os dados considerados (por exemplo, mesmo que se aumente indefinidamente o efetivo policial nas ruas, chegará um momento em que as cidades estarão tão seguras que acréscimos no efetivo não terão grande impacto: haverá então retornos decrescentes de escala). A orientação do modelo, por sua vez, é para outputs. Para Emrouznejad e Amin (2009), neste caso o numerador da variável-razão deve ser incluído como output e o denominador como input.

\subsection{Escolha das variáveis após considerações de convexidade}

As ressalvas de Emrouznejad e Amin (2009) sobre a convexidade de um modelo DEA na presença de variáveis-razão levam à seguinte reorganização das variáveis, indicada na Tabela4: 
Tabela 4 - Definição das variáveis do modelo DEA após considerações de convexidade

\begin{tabular}{|c|c|c|c|}
\hline Variável & Nome & Fonte de dados & Cálculo \\
\hline Input 1 & $\begin{array}{l}\text { Despesas } \\
\text { Totais }\end{array}$ & $\begin{array}{l}\text { ABSP 2017, Tabela } 26 \text { - Despesas } \\
\text { realizadas com a Função Segurança } \\
\text { Pública, por Subfunção }\end{array}$ & $\begin{array}{l}\text { Despesas realizadas com a Função } \\
\text { Segurança Pública (Total) }\end{array}$ \\
\hline Input 2 & $\begin{array}{l}\text { Efetivo } \\
\text { Total }\end{array}$ & $\begin{array}{c}\text { ABSP 2017, Tabela } 25 \text { - Efetivo } \\
\text { fixado e existente das Polícias } \\
\text { Militar e Civis }\end{array}$ & $\begin{array}{c}\text { Efetivo existente da Polícia Militar + } \\
\text { Efetivo existente da Polícia Civil }\end{array}$ \\
\hline Input 3 & CVLI & $\begin{array}{c}\text { ABSP 2017, Tabela } 01 \text { - Mortes } \\
\text { violentas intencionais }\end{array}$ & $\begin{array}{c}\text { Total de crimes CVLI (Homicídio } \\
\text { Doloso + Latrocínio + Lesão Corporal } \\
\text { Seguida de Morte) }\end{array}$ \\
\hline Output 1 & População & IBGE & População de acordo com IBGE \\
\hline
\end{tabular}

Fonte: Os autores.

Percebe-se que a razão População/(No.CVLI), output original, foi dividida em duas variáveis adicionais: CVLI (denominador) como o Input 3, e População (numerador) como o novo Output 1. Dessa forma, a função-objetivo a ser maximizada pode ser escrita de acordo com a equação 2 abaixo:

$$
\max h_{0}=\frac{u_{1} \text { População }}{v_{1} \text { Despesas }+v_{2} \text { Efetivo }+v_{3} C V L I}
$$

Em que $u_{1}$ é o peso do output "População", e $v_{1}, v_{2}$ e $v_{3}$ são os pesos dos inputs "Despesas", "Efetivo" e "CVLI", respectivamente. A maximização do número de habitantes para cada real gasto com segurança, cada funcionário policial e cada crime praticado é exatamente equivalente à minimização das despesas, efetivo e CVLI por habitante, respectivamente.

\subsection{Teste da regra de ouro}

A quantidade mínima de DMUs que um modelo DEA com 3 inputs e 1 output deve apresentar para manter seu poder discriminatório é dada segundo a equação 3 (Serrano, Martínez, \&Munõz,2016):

$$
n \geq \max \{3(m+s), m \times s\}
$$

em que $n$ é o número de DMUs, $m$ o número de inputs e $s$ o número de outputs. Neste estudo, tem-se $n \geq \max \{3(3+1), 3 \times 1\}$, logo deve-se ter $n \geq 12$. Como as DMUs correspondem aos 27 estados brasileiros, $\log 0 n=27 \geq 12$ e o modelo obedece à regra de ouro.

\subsection{Dados utilizados no modelo}

A Tabela 5 a seguir reúne dados utilizados no modelo BCC para cálculo das eficiências de serviços de segurança pública no Brasil. 
Tabela 5 - Dados utilizados no modelo

DEA - Avaliação da Segurança Pública no Brasil

\begin{tabular}{|c|c|c|c|c|}
\hline \multirow{2}{*}{ UF } & Input 1 & Input 2 & Input 3 & Output 1 \\
\hline & Despesas & Efetivo & CVLI & População \\
\hline Acre & 43,42 & 35,21 & 3,68 & 8,17 \\
\hline Alagoas & 104,22 & 92,63 & 17,69 & 33,59 \\
\hline Amapá & 41,40 & 47,83 & 3,29 & 7,82 \\
\hline Amazonas & 139,77 & 116,23 & 11,40 & 40,02 \\
\hline Bahia & 414,64 & 372,78 & 66,53 & 152,77 \\
\hline Ceará & 190,60 & 186,21 & 34,57 & 89,64 \\
\hline Distrito Federal & 70,74 & 180,15 & 6,52 & 29,77 \\
\hline Espírito Santo & 130,99 & 123,58 & 12,46 & 39,74 \\
\hline Goiás & 301,00 & 163,20 & 27,25 & 66,96 \\
\hline Maranhão & 138,79 & 96,89 & 22,15 & 69,54 \\
\hline Mato Grosso & 181,65 & 111,04 & 11,72 & 33,06 \\
\hline Mato Grosso do Sul & 127,12 & 80,30 & 6,08 & 26,82 \\
\hline Minas Gerais & 888,45 & 511,58 & 43,67 & 209,98 \\
\hline Pará & 222,25 & 183,82 & 39,27 & 82,73 \\
\hline Paraíba & 112,08 & 114,20 & 13,22 & 39,99 \\
\hline Paraná & 381,33 & 237,04 & 26,47 & 112,43 \\
\hline Pernambuco & 231,47 & 246,38 & 44,79 & 94,10 \\
\hline Piauí & 70,06 & 74,42 & 7,04 & 32,12 \\
\hline Rio de Janeiro & 915,98 & 582,39 & 53,37 & 166,36 \\
\hline Rio Grande do Norte & 105,85 & 105,18 & 19,11 & 34,75 \\
\hline Rio Grande do Sul & 333,61 & 217,53 & 30,38 & 112,87 \\
\hline Rondônia & 76,20 & 75,70 & 5,86 & 17,87 \\
\hline Roraima & 35,62 & 24,40 & 0,97 & 5,14 \\
\hline Santa Catarina & 218,15 & 126,09 & 9,76 & 69,11 \\
\hline São Paulo & $1.099,45$ & $1.203,61$ & 40,69 & 447,50 \\
\hline Sergipe & 82,06 & 63,35 & 13,55 & 22,66 \\
\hline Tocantins & 73,15 & 54,55 & 4,00 & 15,33 \\
\hline
\end{tabular}

Fonte: Dados de ABSP (2017) e IBGE(2017).

As unidades dos dados encontrados nas respectivas fontes foram ajustadas pois, em se tratando de cálculos computacionais, a presença simultânea de números muito grandes e muito pequenos pode causar problemas de escala, onde perde-se a precisão dos resultados a cada operação devido a sucessivos arredondamentos. Na Tabela 6 são relacionadas as unidades das variáveis fornecidas na fonte e aquelas utilizadas no modelo. 
Tabela 6 - Equivalência de unidades na fonte dos dados e modelo

\begin{tabular}{lll}
\multicolumn{1}{c}{ Variável } & \multicolumn{2}{c}{ Fonte } \\
& \multicolumn{1}{c}{ Unidade } \\
\hline Despesas & R\$ Correntes & R\$ Correntes $\times 10^{-7}$ \\
Efetivo & No. Agentes & No. Agentes $\times 10^{-2}$ \\
CVLI & No. CVLI & No. CVLI $\times 10^{-2}$ \\
População & No. Habitantes & No. Habitantes $\times 10^{-5}$
\end{tabular}

Fonte: Os autores.

Cabe salientar que para a DEA, a unidade das variáveis é irrelevante, contanto que as mesmas unidades sejam usadas para todas as DMUs. $O$ ajuste dos valores conforme a Tabela 6 equivale a uma transformação de unidades e não afetará os resultados do modelo. O software escolhido para resolução do modelo proposto foi o Open Source Data Envelopment Graphical User Interface (OSDEAGUI, 2018). O modelo adotado foi de retornos variáveis de escala (BCC) e orientação para outputs.

\section{Resultados e discussão}

\subsection{Aplicação do modelo BCC}

A Tabela 7 exibe as eficiências dos serviços de segurança pública dos estados brasileiros.

Tabela 7 - Eficiência dos serviços de segurança pública dos estados brasileiros

\begin{tabular}{lc}
\hline UF & Eficiência \\
\hline Santa Catarina & $100 \%$ \\
Piauí & $100 \%$ \\
Roraima & $100 \%$ \\
São Paulo & $100 \%$ \\
Rio Grande do Sul & $100 \%$ \\
Maranhão & $100 \%$ \\
Ceará & $99,68 \%$ \\
Minas Gerais & $98,74 \%$ \\
Distrito Federal & $96,16 \%$ \\
Paraná & $95,21 \%$ \\
Bahia & $92,80 \%$ \\
Pernambuco & $88,77 \%$ \\
Pará & $83,21 \%$ \\
Amapá & $80,89 \%$ \\
Paraíba & $75,90 \%$ \\
Acre & $73,29 \%$ \\
Goiás & $71,73 \%$ \\
Rio de Janeiro & $70,29 \%$ \\
Amazonas & $69,97 \%$
\end{tabular}




\begin{tabular}{lc}
\hline UF & Eficiência \\
\hline Tocantins & $68,01 \%$ \\
Mato Grosso do Sul & $67,76 \%$ \\
Espírito Santo & $67,75 \%$ \\
Rio Grande do Norte & $67,34 \%$ \\
Alagoas & $66,22 \%$ \\
Sergipe & $63,88 \%$ \\
Rondônia & $58,38 \%$ \\
Mato Grosso & $52,23 \%$ \\
Média & $81,79 \%$ \\
\hline
\end{tabular}

Fonte: Os autores.

Os estados identificados como $100 \%$ eficientes pelo modelo foram Santa Catarina, Piauí, Roraima, São Paulo, Rio Grande do Sul e Maranhão. Estes 6 estados, portanto, formam a fronteira de eficiência e servem como benchmarks para os demais. Em contrapartida, os estados que demonstraram o pior desempenho nacional foram o Mato Grosso e Rondônia, com escores de eficiência abaixo dos 60\%. Estabelecendo-se faixas de eficiência, onde um escore de até $70 \%$ é considerado de baixa eficiência, de 70-90\% de eficiência moderada, e de 90-99,99\% de alta eficiência, é possível resumir os resultados de acordo com a Tabela 8:

Tabela 8 - Faixas de eficiência dos estados

\begin{tabular}{cccc}
\hline Eficiência & Categoria & Estados & \% Estados \\
\hline$<70 \%$ & Baixa & MT, RO, SE, AL, RN, ES, MS, TO, AM & $33,33 \%$ \\
$70-90 \%$ & Moderada & RJ, GO, AC, PB, AP, PA, PE & $25,93 \%$ \\
$90-99,99 \%$ & Alta & BA, PR, DF, MG, CE & $18,52 \%$ \\
$100 \%$ & Benchmark & MA, RS, SP, RR, PI, SC & $22,22 \%$ \\
\hline
\end{tabular}

Fonte: Os autores.

Na Figura 3 é apresentado um mapa dos estados brasileiros segundo as faixas de eficiência em que foram classificados. 
Figura 3 - Mapa dos escores de eficiência dos serviços de segurança pública dos estados

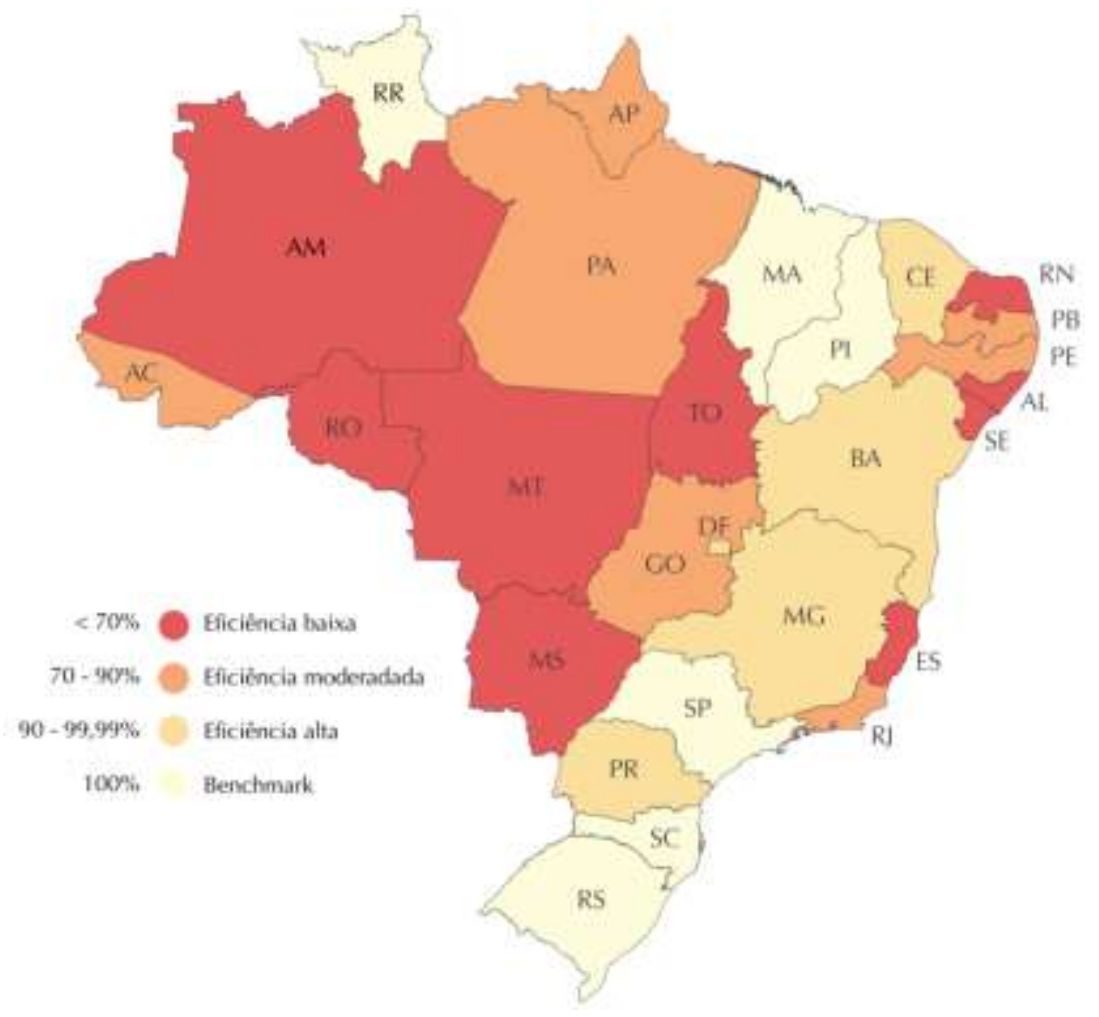

Fonte: Os autores.

Percebe-se que, sem grande rigor, há um agrupamento geográfico das unidades com escores de eficiência insatisfatórios (<90\%) e das unidades com escores satisfatórios (>90\%). Isto pode sugerir a presença de fatores relacionados à segurança semelhantes entre estes estados. Esta hipótese, no entanto, requer investigações ulteriores.

Note que as eficiências encontradas são no geral altas, com média nacional de 81,79\%, o que poderia induzir o leitor à conclusão de que os serviços de segurança oferecidos pelos estados são na maior parte dos casos eficientes e, por conseguinte, que os estados são na verdade seguros. Para interpretar as eficiências mais altas do que o esperado, é importante lembrar que a DEA é uma técnica de análise relativa, quer dizer, avalia as DMUs apenas umas contra as outras e não necessariamente contra as melhores práticas mundiais. Das 50 cidades mais violentas do mundo em 2016, 19 eram brasileiras, distribuídas entre 17 estados (Consejo Ciudadano para la Seguridad Pública y la Justicia Penal, 2017). Isto significa que 17 das 27, ou 63\%, das DMUs analisadas figuraram entre os piores resultados mundiais naquele ano. Em vista disto, pode-se estabelecer que as altas eficiências vistas na Tabela 7 são relativas a uma amostra de baixa performance no contexto global e não podem ser entendidas diretamente como um bom resultado.

Ademais, deve-se levar em consideração a confiabilidade dos dados utilizados como variáveis do modelo. Todos os dados usados como inputs, retirados do ABSP (2017), estão sujeitos à classificação dos estados em grupos segundo qualidade de informações (vide Tabela 2). Os estados 
que apresentam níveis menores de confiança podem, assim, distorcer as eficiências calculadas. O estado da Bahia, por exemplo, aparece com eficiência de 92,80\%, mas pertence ao Grupo 4 (o pior) de qualidade de dados, de forma que não há como assegurar que este índice corresponda ao seu real desempenho.

\subsection{Projeções na fronteira de eficiência}

A DEA permite identificar o quanto cada unidade precisa melhorar para se tornar eficiente. Dado que o modelo em estudo é orientado a outputs não serão avaliadas as projeções dos inputs já que a proposta é que estes sejam mantidos inalterados. Porém, uma vez que o output original era uma razão (Tabela 3), tendo sido necessário desagregá-lo em seu numerador (Input 3) e denominador (Output 1) a fim de se respeitar o axioma de convexidade da DEA, tem-se que um dos inputs faz parte da solução. Neste momento de avaliação de resultados, torna-se então conveniente agregar novamente a fração. Os valores do output-razão, designado aqui como Output ${ }^{*}$, foram obtidos através dos seguintes quocientes para os dados de entrada do modelo (antes do cálculo da eficiência, Equação 21) e para sua projeção na fronteira de eficiência (depois do cálculo da eficiência, Equação 22):

$$
\begin{gathered}
\text { Output } 1_{E}^{*}=\frac{\text { Output } 1}{\text { Input } 3} \\
\text { Output } 1_{P}^{*}=\frac{\text { ProjeçãodoOutput } 1}{\text { Input } 3}
\end{gathered}
$$

Nota-se que a projeção do Output 1* (Equação 4) foi obtida dividindo-se a projeção do Output 1 pelo Input 3 de entrada, respeitando-se a inalterabilidade dos inputs conforme orientação do modelo. Na Tabela 9 são apresentados os números encontrados.

Tabela 9 - Metas para as taxas de habitante / CVLI

\begin{tabular}{lccc|cc}
\hline & \multicolumn{3}{c|}{ Dados de entrada } & \multicolumn{2}{c}{ Projeção } \\
\cline { 2 - 6 } UF & Input 3 & Output 1 & Output 1* & Output 1 & Output 1* \\
\cline { 2 - 6 } & CVLI & População & Hab / & População x & Hab / \\
& $\times 10^{-2}$ & $\times 10^{-5}$ & CVLI & $10^{-5}$ & CVLI \\
\hline Acre & 3,68 & 8,17 & $2.219,26$ & 11,14 & $3.028,14$ \\
Alagoas & 17,69 & 33,59 & $1.898,79$ & 50,72 & $2.867,24$ \\
Amapá & 3,29 & 7,82 & $2.377,80$ & 9,67 & $2.939,56$ \\
Amazonas & 11,40 & 40,02 & $3.510,23$ & 57,19 & $5.016,91$ \\
Bahia & 66,53 & 152,77 & $2.296,19$ & 164,62 & $2.474,34$ \\
Ceará & 34,57 & 89,64 & $2.592,90$ & 89,92 & $2.601,22$ \\
Distrito Federal & 6,52 & 29,77 & $4.566,28$ & 30,96 & $4.748,77$ \\
Espírito Santo & 12,46 & 39,74 & $3.189,16$ & 58,65 & $4.707,42$ \\
Goiás & 27,25 & 66,96 & $2.457,19$ & 93,35 & $3.425,84$
\end{tabular}




\begin{tabular}{|c|c|c|c|c|c|}
\hline \multirow{3}{*}{ UF } & \multicolumn{3}{|c|}{ Dados de entrada } & \multicolumn{2}{|c|}{ Projeção } \\
\hline & Input 3 & Output 1 & Output 1* & Output 1 & Output $1^{*}$ \\
\hline & $\begin{array}{l}\text { CVLI } \\
\times 10^{-2}\end{array}$ & $\begin{array}{l}\text { População } \\
\times 10^{-5}\end{array}$ & $\begin{array}{l}\mathrm{Hab} / \\
\mathrm{CVLI}\end{array}$ & $\begin{array}{c}\text { População x } \\
10^{-5}\end{array}$ & $\begin{array}{l}\mathrm{Hab} / \\
\mathrm{CVLI}\end{array}$ \\
\hline Maranhão & 22,15 & 69,54 & $3.139,52$ & 69,54 & $3.139,52$ \\
\hline Mato Grosso & 11,72 & 33,06 & $2.820,42$ & 63,29 & $5.400,12$ \\
\hline Mato Grosso do Sul & 6,08 & 26,82 & $4.411,82$ & 39,59 & $6.510,83$ \\
\hline Minas Gerais & 43,67 & 209,98 & $4.808,23$ & 212,65 & $4.869,58$ \\
\hline Pará & 39,27 & 82,73 & $2.106,63$ & 99,42 & $2.531,63$ \\
\hline Paraíba & 13,22 & 39,99 & $3.025,28$ & 52,70 & $3.986,01$ \\
\hline Paraná & 26,47 & 112,43 & $4.247,34$ & 118,08 & $4.460,89$ \\
\hline Pernambuco & 44,79 & 94,10 & $2.100,99$ & 106,01 & $2.366,71$ \\
\hline Piauí & 7,04 & 32,12 & $4.562,76$ & 32,12 & $4.562,76$ \\
\hline Rio de Janeiro & 53,37 & 166,36 & $3.117,11$ & 236,68 & $4.434,74$ \\
\hline Rio Grande do Norte & 19,11 & 34,75 & $1.818,42$ & 51,60 & $2.700,38$ \\
\hline Rio Grande do Sul & 30,38 & 112,87 & $3.715,11$ & 112,87 & $3.715,11$ \\
\hline Rondônia & 5,86 & 17,87 & $3.049,96$ & 30,61 & $5.224,30$ \\
\hline Roraima & 0,97 & 5,14 & $5.301,33$ & 5,14 & $5.301,33$ \\
\hline Santa Catarina & 9,76 & 69,11 & $7.080,48$ & 69,11 & $7.080,48$ \\
\hline São Paulo & 40,69 & 447,50 & $10.997,71$ & 447,50 & $10.997,71$ \\
\hline Sergipe & 13,55 & 22,66 & $1.672,16$ & 35,47 & $2.617,78$ \\
\hline Tocantins & 4,00 & 15,33 & $3.832,26$ & 22,54 & $5.634,90$ \\
\hline
\end{tabular}

A projeção do Output 1* na fronteira de eficiência representa a maximização da taxa de Habitantes / CVLI, ou a melhor taxa alcançável por cada UF dados os seus recursos Despesas (Input 1) e Efetivo (Input 2) disponíveis. Sendo assim, para os níveis de inputs atuais, as taxas encontradas na Tabela 9 são possíveis de se alcançar, e, portanto, servem como meta para que as unidades tornem-se $100 \%$ eficientes. Percebe-se que os valores das metas para os estados benchmarks são os mesmos dos Outputs $1^{*}$ de entrada, já que estas unidades já se encontram na fronteira de eficiência.

Uma taxa pode ser aumentada de duas maneiras: aumentando-se seu numerador ou diminuindo-se seu denominador. Neste caso o numerador é a população estadual, uma variável com pouquíssima flexibilidade (uma alteração significativa demoraria gerações para acontecer), de maneira que o decréscimo de seu denominador, ou o número de CVLI, seria uma opção mais realista para alcançar-se a taxa máxima possível sugerida pelo modelo.

Desta maneira, na Tabela 10 são indicadas as reduções necessárias no número de CVLI para que cada estado alcance sua melhor "taxa de segurança". Em outras palavras, mantendo-se as despesas e efetivos policiais inalterados, os estados deveriam ser capazes de produzir as taxas projetadas (projeção de Output 1*) através da redução de CVLI na porcentagem indicada. 
Tabela 10 - Metas para redução do número de CVLI

\begin{tabular}{|c|c|c|c|c|c|}
\hline UF & CVLI (2016) & Meta & Meta \% & UF & Meta \% ord. \\
\hline Acre & 368 & 270 & $-27 \%$ & Mato Grosso & $-48 \%$ \\
\hline Alagoas & 1769 & 1171 & $-34 \%$ & Rondônia & $-42 \%$ \\
\hline Amapá & 329 & 266 & $-19 \%$ & Sergipe & $-36 \%$ \\
\hline Amazonas & 1140 & 798 & $-30 \%$ & Alagoas & $-34 \%$ \\
\hline Bahia & 6653 & 6174 & $-7 \%$ & Rio Grande do Norte & $-33 \%$ \\
\hline Ceará & 3457 & 3457 & $0 \%$ & Espírito Santo & $-32 \%$ \\
\hline Distrito Federal & 652 & 627 & $-4 \%$ & Mato Grosso do Sul & $-32 \%$ \\
\hline Espírito Santo & 1246 & 844 & $-32 \%$ & Tocantins & $-32 \%$ \\
\hline Goiás & 2725 & 1955 & $-28 \%$ & Amazonas & $-30 \%$ \\
\hline Maranhão & 2215 & 2215 & $0 \%$ & Rio de Janeiro & $-30 \%$ \\
\hline Mato Grosso & 1172 & 612 & $-48 \%$ & Goiás & $-28 \%$ \\
\hline Mato Grosso do Sul & 608 & 412 & $-32 \%$ & Acre & $-27 \%$ \\
\hline Minas Gerais & 4367 & 4312 & $-1 \%$ & Paraíba & $-24 \%$ \\
\hline Pará & 3927 & 3268 & $-17 \%$ & Amapá & $-19 \%$ \\
\hline Paraíba & 1322 & 1003 & $-24 \%$ & Pará & $-17 \%$ \\
\hline Paraná & 2647 & 2520 & $-5 \%$ & Pernambuco & $-11 \%$ \\
\hline Pernambuco & 4479 & 3976 & $-11 \%$ & Bahia & $-7 \%$ \\
\hline Piauí & 704 & 704 & $0 \%$ & Paraná & $-5 \%$ \\
\hline Rio de Janeiro & 5337 & 3751 & $-30 \%$ & Distrito Federal & $-4 \%$ \\
\hline Rio Grande do Norte & 1911 & 1287 & $-33 \%$ & Minas Gerais & $-1 \%$ \\
\hline Rio Grande do Sul & 3038 & 3038 & $0 \%$ & Ceará & $0 \%$ \\
\hline Rondônia & 586 & 342 & $-42 \%$ & Maranhão & $0 \%$ \\
\hline Roraima & 97 & 97 & $0 \%$ & Rio Grande do Sul & $0 \%$ \\
\hline Santa Catarina & 976 & 976 & $0 \%$ & Piauí & $0 \%$ \\
\hline São Paulo & 4069 & 4069 & $0 \%$ & Roraima & $0 \%$ \\
\hline Sergipe & 1355 & 866 & $-36 \%$ & Santa Catarina & $0 \%$ \\
\hline Tocantins & 400 & 272 & $-32 \%$ & São Paulo & $0 \%$ \\
\hline
\end{tabular}

Fonte: Os autores.

Percebe-se que ordenar os estados pela oportunidade de redução de crimes corresponde exatamente ao inverso de ordená-los de acordo com seu escore de eficiência calculada pelo modelo (Tabela 7), já que na medida em que não aplicam seus recursos de maneira ótima, tornam-se ineficientes. Sendo assim, as UF com maior oportunidade de redução de CVLI são aquelas classificadas como pouco e moderadamente eficientes. Isto fica explícito na Tabela 11, que relaciona as faixas de eficiência dos estados às suas metas de redução do número de CVLI. 
Tabela 11 - Faixas de eficiência e respectivas metas de redução de CVLI

\begin{tabular}{cccc}
\hline Eficiência & Categoria & Estados & Meta Redução CVLI \\
\hline$<70 \%$ & Baixa & MT, RO, SE, AL, RN, ES, MS, TO, AM & $30-48 \%$ \\
$70-90 \%$ & Moderada & RJ, GO, AC, PB, AP, PA, PE & $11-30 \%$ \\
$90-99,99 \%$ & Alta & BA, PR, DF, MG, CE & $0,01-7 \%$ \\
$100 \%$ & Benchmark & MA, RS, SP, RR, PI, SC & $0,00 \%$
\end{tabular}

Fonte: Os autores.

\subsection{Unidades-alvo}

Na Tabela 12 é fornecida uma lista dos estados benchmarks mais próximos de cada estado ineficiente, e são assim chamados de "unidades-alvo". As unidades-alvo são obtidas como saída do modelo DEA. Dessa forma, os estados ineficientes podem olhar para as práticas dos estados benchmarks mais relevantes como guias para alcançar a eficiência, replicando-as em sua própria unidade na medida do possível.

Tabela 12 - Unidades-alvo para os estados ineficientes

\begin{tabular}{|c|c|c|c|c|}
\hline \multirow{2}{*}{$\begin{array}{l}\text { UF } \\
\text { Acre }\end{array}$} & \multicolumn{2}{|l|}{$\leftarrow$ Mais relevante } & \multicolumn{2}{|c|}{ Menos relevante $\rightarrow$} \\
\hline & Roraima & Piauí & Maranhão & \\
\hline Alagoas & Piauí & Maranhão & & \\
\hline Amapá & Roraima & Piauí & & \\
\hline Amazonas & Piauí & Santa Catarina & Maranhão & São Paulo \\
\hline Bahia & Rio Grande do Sul & Maranhão & São Paulo & \\
\hline Ceará & Maranhão & São Paulo & & \\
\hline Distrito Federal & Piauí & Roraima & São Paulo & \\
\hline Espírito Santo & Piauí & Maranhão & São Paulo & $\begin{array}{l}\text { Santa } \\
\text { Catarina }\end{array}$ \\
\hline Goiás & Rio Grande do Sul & Maranhão & & \\
\hline Mato Grosso & Santa Catarina & Maranhão & Piauí & Roraima \\
\hline Mato Grosso do Sul & Santa Catarina & Roraima & Piauí & São Paulo \\
\hline Minas Gerais & Rio Grande do Sul & São Paulo & & \\
\hline Pará & Maranhão & Rio Grande do Sul & São Paulo & \\
\hline Paraíba & Piauí & Maranhão & São Paulo & \\
\hline Paraná & Maranhão & Rio Grande do Sul & São Paulo & \\
\hline Pernambuco & Maranhão & São Paulo & & \\
\hline Rio de Janeiro & Rio Grande do Sul & São Paulo & & \\
\hline Rio Grande do Norte & Maranhão & Piauí & & \\
\hline Rondônia & Piauí & Roraima & Santa Catarina & São Paulo \\
\hline Sergipe & Roraima & Maranhão & Piauí & \\
\hline Tocantins & Roraima & Piauí & Santa Catarina & São Paulo \\
\hline
\end{tabular}

Fonte: Os autores. 


\section{Conclusão}

Este estudo propôs uma avaliação dos serviços de segurança pública do Brasil através da análise por envoltória de dados (DEA). Foram utilizados os dados da edição de 2017 do ABSP, com dados referentes ao ano de 2016, documento que compila e analisa dados de registros policiais sobre criminalidade, informações sobre os sistemas prisionais e gastos com segurança pública e principal fonte de dados relativos à segurança no país, para estruturar o modelo DEA. Para cada unidade federativa, foram selecionados dois inputs: suas despesas totais com segurança no período de 2016, representando os recursos monetários do estado, e seu total de efetivo policial trabalhando para promover a segurança, representando seus recursos humanos. Como output, foi selecionada uma "taxa de segurança” para ser maximizada: a razão No. Habitantes / No. CVLI.

Os resultados mostraram que 6 estados, Santa Catarina, Piauí, Roraima, São Paulo, Rio Grande do Sul e Maranhão foram 100\% eficientes na alocação de suas despesas e efetivo policial a fim de se maximizar a taxa de habitantes / CVLI em 2016. Em contrapartida, os estados que demonstraram o pior desempenho nacional foram o Mato Grosso e Rondônia, com escores de eficiência abaixo dos $60 \%$. Classificando-se os estados em grupos de acordo com seu escore, 33,33\% das unidades federativas apresentaram baixa eficiência (< 70\%) e 25,93\% deles, eficiência moderada (70 - 90\%), totalizando $59,26 \%$ dos estados na faixa de desempenho considerada insatisfatória. A próxima categoria configura o grupo de eficiência alta (90 - 99,99\%), onde estavam presentes 18,52\% das UF. A soma dos estados benchmark com os estados altamente eficientes reuniu $40,74 \%$ dos estados na faixa de desempenho considerada satisfatória.

As eficiências encontradas são no geral altas, o que poderia induzir o leitor à conclusão de que os serviços de segurança oferecidos pelos estados são na maior parte dos casos eficientes. No entanto, é importante enfatizar que a DEA é uma técnica de análise relativa, de forma que os estados considerados eficientes dizem respeito somente ao conjunto de estados comparados. Isso não indica que sejam eficientes se comparados às melhores práticas internacionais. Ademais, deve-se levar em consideração a confiabilidade dos dados de entrada do modelo. Todos os dados retirados do ABSP estão sujeitos à classificação dos estados em grupos segundo qualidade de informações. Os estados que apresentam menores níveis de confiança podem, assim, distorcer as eficiências calculadas, aparecendo como mais eficientes do que deveriam.

Uma contribuição secundária deste estudo foi a demonstração do uso de uma razão como variável de um modelo DEA de maneira a respeitar o axioma de convexidade da técnica, muitas vezes ignorado na literatura.

Finalmente, como extensões a este trabalho sugerem-se o estudo dos estados identificados como $100 \%$ eficientes a fim de se identificarem as melhores práticas do país; e a aplicação de um modelo DEA que compare os serviços de segurança do Brasil com aqueles de países que apresentam baixos índices criminais. 


\section{Referências}

Adler, N., \& Yazhemsky, E. (2010). Improving discrimination in data envelopment analysis: PCA-DEA versus variable reduction. Which method at what cost? European Journal of Operational Research, 202(1), 273-284.

Banker, R.D., Charnes, A, \& Cooper, W.W. (1984). Some models for estimating technical and scale inefficiencies in data envelopment analysis. Management Science, 30(9), 1078-1092.

Besanko, D., \& Braeutigam, R. R. (2011). Microeconomics. 4a. ed., Wiley.

Charnes, A. Cooper, W.W., \& Rhodes, E. (1978). Measuring the efficiency of decision making units. European Journal of Operational Research, 2(6), 429-444.

Colin, E. C. (2007). Pesquisa Operacional: 170 aplicações em estratégia, finanças, logística, produção, marketing e vendas. Rio de Janeiro: LTC.

Consejo Ciudadano para la Seguridad Pública y la Justicia Penal. (2017). Las 50 ciudades más violentas del mundo 2016 + Metodología. México.

Emrouznejad, A., \& Amin, G. R. (2009). DEA models for ratio data: Convexity consideration. Applied Mathematical Modelling, 33(1), 486-498.

Emrouznejad, A., Banker, R., Lopes, A. L. M., \& Almeida, M. R. (2013). Data envelopment analysis in the public sector. Socio-Economic Planning Sciences, 48, 2-3.

Fernandes, M. D. (2016). A. Análise de eficiência da segurança pública dos estados brasileiros no ano de 2014. Monografia, Universidade Federal do Rio Grande do Norte, Natal, RN, Brasil.

Fórum Brasileiro de Segurança Pública (2017). Anuário Brasileiro de Segurança Pública. São Paulo, SP: Autor.

Fioretti, G. (2008). The Production Function. Bolonha, Itália: University of Bologna.

Hollingsworth, B., \& Smith, P. (2003). Use of ratios in data envelopment analysis. Applied Economics Letters, 10(11), 733-735.

Lima, F., \& Marinho, E. L. L. (2016). Segurança pública no Brasil: eficiência e defasagem tecnológica. Anais do XLII Encontro Nacional de Economia, Natal, RN, Brasil.

Mello, J. C. C. B. S. D., Meza, L. A., Gomes, E. G., \& Neto, L. B. (2005). Curso de Análise de Envoltória de Dados. XXXVII Simpósio Brasileiro de Pesquisa Operacional, Gramado, RS, Brasil.

Observatório de Segurança Pública (2018). A segurança pública no Brasil. Recuperado em 30 de Maio de 2018 de http://www.observatoriodeseguranca.org/seguranca.

OSDEA-GUI (2018). Open source data envelopment graphical user interface. Recuperado em 30 de Maio 2018 de http://opensourcedea.org/osdea-gui/.

Paulo, J. C. D. (2016). Eficiência da aplicação de recursos públicos em segurança nos estados brasileiros. Monografia, Universidade Federal de Alfenas, Varginha, MG, Brasil.

Pellegrini, F. R. (2000). Metodologia para implementação de sistemas de previsão de demanda. Dissertação, Universidade Federal do Rio Grande do Sul, Porto Alegre, RS, Brasil. 
Schull, A. N., Feitósa, C. G., \& Hein, A. F. (2014). Análise da eficiência dos gastos em segurança pública nos estados brasileiros através da Análise Envoltória de Dados (DEA). Revista Capital Científico Eletrônica, 12(3).

Serrano, V. O., Martínez, P. L., \& Munõz, J. P. A. (2016). Measuring the technical efficiency of public service broadcasters: an application of DEA in Spain. Revista de Métodos Cuantitativos para la Economía y la Empresa,21, 5-20.

Recebido em: 14 nov. 2018 / Aprovado em: 08 maio 2019

Para referenciar este texto

American Psychological Association (APA)

Almeida, I. S., \& Pitombeira, A. R. Neto. (2020). Eficiência dos serviços de segurança pública no Brasil: uma análise por envoltória de dados. Exacta, 18(3), 540-560. https://doi.org/10.5585/ExactaEP.v18n3.10928. 